\title{
Theoretical Study on the Photosensitizer Mechanism of Phenalenone in aqueous and lipid media
}

\author{
Cesar Espinoza, ${ }^{\dagger}$ Ángel Trigos, ${ }^{\dagger}$ Manuel E. Medina ${ }^{*}+$ \\ ${ }^{\dagger}$ Laboratorio de Alta Tecnología de Xalapa, Universidad Veracruzana, Calle Medicos 5, Col. \\ Unidad del Bosque 91010, Xalapa, Veracruz, México. \\ †Centro de Investigaciones Biomédicas, Universidad Veracruzana, Dr. Luis Castelazo Ayala s/n, \\ Col. Industrial las Animas 91190, Xalapa, Veracruz, México.
}

Optimized geometries of neutral, excited, oxidized and reduced phenalenone.

Aqueous media

* To whom correspondence should be addresed. E-mail: manmedina@uv.mx 


$$
\begin{aligned}
& { }^{1} \mathrm{PN} \\
& \mathrm{E}_{0}=-575.187912 \\
& \mathrm{H}=-575.177596 \\
& \mathrm{G}=-575.222651 \\
& \mathrm{~T} 1=0.014 \\
& 0,1
\end{aligned}
$$

\begin{tabular}{|c|c|c|c|}
\hline $\mathrm{C}$ & -1.755438354 & -1.9207049395 & 0.0001132278 \\
\hline $\mathrm{C}$ & -0.472714989 & -1.408000637 & -0.0000113532 \\
\hline $\mathrm{C}$ & -0.277113509 & 0.0010238494 & 0.0000362284 \\
\hline $\mathrm{C}$ & -1.4022516122 & 0.8617970637 & 0.0002136863 \\
\hline $\mathrm{C}$ & -2.7059617965 & 0.2963657702 & 0.0003256451 \\
\hline $\mathrm{C}$ & -2.8770867168 & -1.0653717662 & 0.0002776732 \\
\hline $\mathrm{H}$ & -1.8966220368 & -2.9963980671 & 0.0000827871 \\
\hline $\mathrm{C}$ & 1.0305604777 & 0.5541833858 & -0.0000303913 \\
\hline $\mathrm{C}$ & -1.1949351647 & 2.2618572873 & 0.0002824164 \\
\hline $\mathrm{H}$ & -3.5621315135 & 0.9624210202 & 0.0004514239 \\
\hline $\mathrm{H}$ & -3.8735733233 & -1.4897497139 & 0.0003579514 \\
\hline $\mathrm{C}$ & 0.0787943913 & 2.783666234 & 0.0001926224 \\
\hline $\mathrm{C}$ & 1.1933801823 & 1.9274083698 & -0.0000106188 \\
\hline $\mathrm{H}$ & -2.05984447 & 2.9168947228 & 0.0003843251 \\
\hline $\mathrm{H}$ & 0.2288748315 & 3.8562480013 & 0.0002654322 \\
\hline $\mathrm{H}$ & 2.1944108549 & 2.3431237216 & -0.000041238 \\
\hline $\mathrm{C}$ & 0.6922028506 & -2.2717708388 & -0.0001510024 \\
\hline $\mathrm{H}$ & 0.5202108191 & -3.3438878069 & -0.000167041 \\
\hline $\mathrm{C}$ & 2.210144731 & -0.3441424236 & -0.00014423 \\
\hline
\end{tabular}




\begin{tabular}{|l|l|l|l|}
\hline $\mathrm{C}$ & 1.9478213621 & -1.7834431345 & -0.0002269245 \\
\hline $\mathrm{H}$ & 2.8129878059 & -2.4363736942 & -0.0004206559 \\
\hline $\mathrm{O}$ & 3.3627891792 & 0.0951275957 & -0.0008439643 \\
\hline
\end{tabular}

$$
\begin{aligned}
& { }^{1} \mathrm{PN}^{*} \\
& \mathrm{E}_{0}=-575.082305 \\
& \mathrm{H}=-575.071727 \\
& \mathrm{G}=-575.117062 \\
& \mathrm{~T} 1=0.017 \\
& 0,1
\end{aligned}
$$

\begin{tabular}{|l|l|l|l|}
\hline C & -1.7963849817 & -1.9546147084 & 0.0001232189 \\
\hline C & -0.4586847266 & -1.4350088542 & -0.0000076054 \\
\hline C & -0.2578924123 & -0.0002661948 & 0.0000486438 \\
\hline C & -1.3951045891 & 0.8681608602 & 0.0002365605 \\
\hline C & -2.6752517096 & 0.3040207579 & 0.0003650914 \\
\hline H & -2.8662242601 & -1.1027765413 & 0.0003102271 \\
\hline C & -1.9337549758 & -3.0295384982 & 0.0000698786 \\
\hline C & 1.0298839826 & 0.5456844013 & -0.0000847143 \\
\hline H & -1.1922627874 & 2.2719107983 & 0.0002756483 \\
\hline H & -3.5400129367 & 0.9593078356 & 0.0005066245 \\
\hline C & -3.8761549452 & -1.4941798212 & 0.0004170039 \\
\hline C & 0.0954829768 & 2.79370686 & 0.0001256703 \\
\hline H & 1.2004591469 & 1.953180251 & -0.0000503328 \\
\hline H & -2.0577958705 & 2.9247497184 & 0.000421089 \\
\hline H & 0.237299786 & 3.8680576112 & 0.0001547404 \\
\hline C & 2.2046092368 & 2.3580065414 & -0.0001536266 \\
\hline
\end{tabular}




\begin{tabular}{|l|l|l|l|}
\hline $\mathrm{H}$ & 0.50621522 & -3.3552056585 & -0.0002594954 \\
\hline $\mathrm{C}$ & 2.1938524406 & -0.3447273514 & -0.0002473443 \\
\hline $\mathrm{C}$ & 1.9442962008 & -1.7596146949 & -0.0003416067 \\
\hline $\mathrm{H}$ & 2.8082573481 & -2.4153401023 & -0.0004913333 \\
\hline $\mathrm{O}$ & 3.3637383482 & 0.1268858371 & -0.000278388 \\
\hline
\end{tabular}

$$
\begin{aligned}
& { }^{3} \mathrm{PN} \\
& \mathrm{E}_{0}=-575.115700 \\
& \mathrm{H}=-575.105145 \\
& \mathrm{G}=-575.151473 \\
& \mathrm{~T} 1=0.033 \\
& 0,3
\end{aligned}
$$

\begin{tabular}{|l|l|l|l|}
\hline C & -1.8032934793 & -1.9528280726 & 0.0001217825 \\
\hline C & -0.4489245207 & -1.4275807777 & -0.0000239551 \\
\hline C & -0.2554905368 & 0.0063577315 & 0.0000269173 \\
\hline C & -1.3933366488 & 0.8718108624 & 0.000221336 \\
\hline C & -2.6902338739 & 0.3003009103 & 0.0003495457 \\
\hline H & -2.8702558422 & -1.1090990074 & 0.000294654 \\
\hline C & -1.9323324701 & -3.0285771701 & 0.0000878468 \\
\hline C & 1.033909554 & 0.5530705155 & -0.0001047478 \\
\hline H & -1.1897731577 & 2.2609720742 & 0.000282405 \\
\hline H & -3.5531941322 & 0.9552388915 & 0.000496462 \\
\hline C & -3.8777745751 & -1.5078991982 & 0.0004007136 \\
\hline C & 0.1056663137 & 2.7901071174 & 0.0001531578 \\
\hline H & 1.2082071894 & 1.9598256233 & -0.0000371613 \\
\hline H & -2.0516266077 & 2.9185364919 & 0.0004337985 \\
\hline & 0.2403700082 & 3.8655183929 & 0.000202745 \\
\hline
\end{tabular}




\begin{tabular}{|l|l|l|l|}
\hline $\mathrm{H}$ & 2.2117281648 & 2.3656440699 & -0.0001403758 \\
\hline $\mathrm{C}$ & 0.6541233468 & -2.2765529681 & -0.0001877061 \\
\hline $\mathrm{H}$ & 0.4999320021 & -3.3491403006 & -0.0001987704 \\
\hline $\mathrm{C}$ & 2.1890186181 & -0.3434833933 & -0.0003235693 \\
\hline $\mathrm{C}$ & 1.9427230409 & -1.7544392571 & -0.0003233566 \\
\hline $\mathrm{H}$ & 2.8083223125 & -2.4074331672 & -0.0004412678 \\
\hline $\mathrm{O}$ & 3.3667392942 & 0.1099246315 & -0.0003544539 \\
\hline
\end{tabular}

$$
\begin{aligned}
& \mathrm{PN}^{\bullet} \\
& \mathrm{E}_{0}=-575.315058 \\
& \mathrm{H}=-575.304725 \\
& \mathrm{G}=-575.350262 \\
& \mathrm{~T} 1=0.017 \\
& -1,2
\end{aligned}
$$

\begin{tabular}{|l|l|l|l|}
\hline C & -1.7877841633 & -1.9391734429 & 0.0001249896 \\
\hline C & -0.4736346981 & -1.427859893 & -0.000009993 \\
\hline C & -0.2713826293 & -0.0126718364 & 0.0000413676 \\
\hline C & -1.4059490669 & 0.8591656498 & 0.0002275551 \\
\hline C & -2.7067958014 & 0.2970542768 & 0.0003571304 \\
\hline C & -2.8828412966 & -1.0804023666 & 0.0003044245 \\
\hline H & -1.9328019284 & -3.0143900841 & 0.0000856381 \\
\hline C & 1.0450384166 & 0.5358269883 & -0.0000873491 \\
\hline C & -1.1918113521 & 2.2605659853 & 0.0002749211 \\
\hline H & -3.5636925664 & 0.9620102917 & 0.0004986415 \\
\hline H & -3.8862014245 & -1.49223402 & 0.0004070834 \\
\hline C & 0.094322037 & 2.7772697725 & 0.0001426042 \\
\hline C & 1.2048074494 & 1.9350745659 & -0.0000339448 \\
\hline
\end{tabular}




\begin{tabular}{|l|l|l|l|}
\hline $\mathrm{H}$ & -2.0525640947 & 2.9208140245 & 0.0004145805 \\
\hline $\mathrm{H}$ & 0.23901181 & 3.8523452673 & 0.0001805422 \\
\hline $\mathrm{H}$ & 2.2041640276 & 2.3520202793 & -0.00012827 \\
\hline $\mathrm{C}$ & 0.6740333264 & -2.267459295 & -0.0001960924 \\
\hline $\mathrm{H}$ & 0.5266284113 & -3.3431918814 & -0.0002390589 \\
\hline $\mathrm{C}$ & 2.2058979369 & -0.3447804344 & -0.000262998 \\
\hline $\mathrm{C}$ & 1.9477509745 & -1.7470544027 & -0.0003198984 \\
\hline $\mathrm{H}$ & 2.8079452588 & -2.409392273 & -0.0004623022 \\
\hline $\mathrm{O}$ & 3.4003633734 & 0.1267368283 & -0.0003835714 \\
\hline
\end{tabular}

$$
\begin{aligned}
& \mathrm{PN}^{\bullet+} \\
& \mathrm{E}_{0}=-574.956810 \\
& \mathrm{H}=-574.946272 \\
& \mathrm{G}=-574.992336 \\
& \mathrm{~T} 1=0.038 \\
& 1,2
\end{aligned}
$$

\begin{tabular}{|l|l|l|l|}
\hline $\mathrm{C}$ & -1.7711307821 & -1.9325007784 & 0.0001148926 \\
\hline $\mathrm{C}$ & -0.4526184762 & -1.4089376724 & -0.0000165964 \\
\hline $\mathrm{C}$ & -0.2587275307 & 0.0134585133 & 0.0000365546 \\
\hline $\mathrm{C}$ & -1.3865969131 & 0.8693192928 & 0.000224782 \\
\hline $\mathrm{C}$ & -2.6777550162 & 0.300209192 & 0.0003492059 \\
\hline $\mathrm{C}$ & -2.8622803845 & -1.093079987 & 0.0002897344 \\
\hline $\mathrm{H}$ & -1.8995676637 & -3.0089301793 & 0.0000732254 \\
\hline $\mathrm{C}$ & 1.0260329437 & 0.563955702 & -0.0001041682 \\
\hline $\mathrm{C}$ & -1.1975375667 & 2.2647930144 & 0.0002895913 \\
\hline $\mathrm{H}$ & -3.5402861336 & 0.9581136371 & 0.0004907589 \\
\hline $\mathrm{H}$ & -3.8658623872 & -1.4977117531 & 0.000389863 \\
\hline
\end{tabular}




\begin{tabular}{|l|l|l|l|}
\hline C & 0.0844097928 & 2.7971862468 & 0.000161228 \\
\hline C & 1.1909435527 & 1.9521684348 & -0.0000364402 \\
\hline $\mathrm{H}$ & -2.0668986519 & 2.9128856831 & 0.0004379593 \\
\hline $\mathrm{H}$ & 0.2260071069 & 3.8701703769 & 0.0002126806 \\
\hline $\mathrm{H}$ & 2.193792371 & 2.3631255847 & -0.0001449576 \\
\hline $\mathrm{C}$ & 0.6710803467 & -2.2833595383 & -0.0001784963 \\
\hline $\mathrm{H}$ & 0.4914465276 & -3.3525506557 & -0.000185804 \\
\hline $\mathrm{C}$ & 2.1985680187 & -0.3425099564 & -0.0003384274 \\
\hline $\mathrm{C}$ & 1.9393122818 & -1.7905723536 & -0.0003180441 \\
\hline $\mathrm{H}$ & 2.8069572189 & -2.4396229032 & -0.000428815 \\
\hline $\mathrm{O}$ & 3.3452153453 & 0.0846640996 & -0.0003827269 \\
\hline
\end{tabular}

Lipid media

$$
\begin{aligned}
& { }^{1} \mathrm{PN} \\
& \mathrm{E}_{0}=-575.193071 \\
& \mathrm{H}=-575.182793 \\
& \mathrm{G}=-575.227747 \\
& \mathrm{~T} 1=0.014 \\
& 0,1
\end{aligned}
$$

\begin{tabular}{|l|l|l|l|}
\hline C & -1.7542092266 & -1.9199124644 & 0.0000970419 \\
\hline C & -0.4733175391 & -1.4082224669 & -0.0000372852 \\
\hline C & -0.2792350714 & 0.002727591 & 0.000013464 \\
\hline C & -1.4046044281 & 0.8631771187 & 0.0002229956 \\
\hline C & -2.7081780572 & 0.2965451636 & 0.000353094 \\
\hline C & -2.8773418393 & -1.0641998373 & 0.0002872472 \\
\hline H & -1.8985437963 & -2.9953293979 & 0.0000648934 \\
\hline
\end{tabular}




\begin{tabular}{|l|l|l|l|}
\hline C & 1.0282078744 & 0.5565449701 & -0.0001441629 \\
\hline C & -1.1955393425 & 2.2639821786 & 0.0003007718 \\
\hline H & -3.5660676121 & 0.9608209109 & 0.0005069546 \\
\hline H & -3.8731986938 & -1.4906062427 & 0.000394931 \\
\hline C & 0.0771190502 & 2.784560382 & 0.0001767632 \\
\hline H & 1.1926147308 & 1.92660898857 & -0.000046266 \\
\hline H & -2.0593186135 & 2.9208491194 & 0.0004646484 \\
\hline H & 0.2277815631 & 3.8573546613 & 0.0002377673 \\
\hline C & 2.1980453098 & 2.3324652292 & -0.000171079 \\
\hline H & 0.6960241361 & -2.2728475694 & -0.0001751281 \\
\hline C & 0.5227864178 & -3.3450691494 & -0.0001348409 \\
\hline C & 2.2190196016 & -0.3401175967 & -0.0004649138 \\
\hline H & 1.949360619 & -1.7870240846 & -0.0003240344 \\
\hline O & 2.8146097745 & -2.440003538 & -0.0003826011 \\
\hline
\end{tabular}

$$
\begin{aligned}
& { }^{1} \mathrm{PN}^{*} \\
& \mathrm{E}_{0}=-575.079976 \\
& \mathrm{H}=-575.069278 \\
& \mathrm{G}=-575.114904 \\
& \mathrm{~T} 1=0.016 \\
& 0,1
\end{aligned}
$$

\begin{tabular}{|l|l|l|l|}
\hline C & -1.7969585892 & -1.956328987 & 0.0001210818 \\
\hline C & -0.4611624915 & -1.4370786424 & -0.0000044604 \\
\hline C & -0.2594365874 & 0.0002836704 & 0.000050611 \\
\hline C & -1.3992578775 & 0.8691260199 & 0.0002380732 \\
\hline C & -2.6756394914 & 0.3037252263 & 0.0003584697 \\
\hline
\end{tabular}




\begin{tabular}{|l|l|l|l|}
\hline C & -2.8673546901 & -1.1051663638 & 0.0003031488 \\
\hline H & -1.9361809075 & -3.0313620607 & 0.0000723423 \\
\hline C & 1.0256359077 & 0.5452645172 & -0.0000761339 \\
\hline C & -1.1914649375 & 2.2754500361 & 0.0002779955 \\
\hline H & -3.5418284894 & 0.9574284198 & 0.0004957576 \\
\hline C & -3.877454097 & -1.4970399481 & 0.0004039319 \\
\hline C & 0.0954974765 & 2.7948090937 & 0.0001399048 \\
\hline H & 1.1999073355 & 1.952219703 & -0.0000355169 \\
\hline H & -2.0546433953 & 2.9316886639 & 0.0004162558 \\
\hline H & 0.2392802729 & 3.8692321459 & 0.0001687662 \\
\hline C & 2.2095793773 & 2.3437208311 & -0.0001435451 \\
\hline H & 0.6617480525 & -2.2799159961 & -0.0001932637 \\
\hline C & 0.5092551465 & -3.3539426409 & -0.0002366315 \\
\hline C & 2.2045462069 & -0.3364955991 & -0.0002661013 \\
\hline H & 1.9459764944 & -1.7603587227 & -0.0003313991 \\
\hline O & 2.8096286931 & -2.4166713169 & -0.0004758346 \\
\hline & 3.3548305905 & 0.1316859755 & -0.000347452 \\
\hline
\end{tabular}

$$
\begin{aligned}
& { }^{3} \mathrm{PN} \\
& \mathrm{E}_{0}=-575.121006 \\
& \mathrm{H}=-575.110466 \\
& \mathrm{G}=-575.156765 \\
& \mathrm{~T} 1=0.033 \\
& 0,3
\end{aligned}
$$

\begin{tabular}{|l|l|l|l|}
\hline $\mathrm{C}$ & -1.8054198389 & -1.9522764363 & 0.0001094549 \\
\hline $\mathrm{C}$ & -0.4485829442 & -1.428618273 & -0.000046304 \\
\hline $\mathrm{C}$ & -0.2566489209 & 0.0047165667 & 0.0000094224 \\
\hline
\end{tabular}




\begin{tabular}{|l|l|l|l|}
\hline C & -1.3957163845 & 0.8710353066 & 0.0002203739 \\
\hline C & -2.6924022283 & 0.2999736264 & 0.0003520286 \\
\hline C & -2.8725289767 & -1.1087351362 & 0.0002921192 \\
\hline C & -1.9376382437 & -3.0279921712 & 0.000083675 \\
\hline C & 1.032013125 & 0.5507380161 & -0.0001293867 \\
\hline H & -1.1893764946 & 2.2593409363 & 0.0002918855 \\
\hline H & -3.5553971974 & 0.95525119 & 0.0005081448 \\
\hline C & -3.8800645605 & -1.5084981473 & 0.0004066905 \\
\hline C & 0.1071368836 & 2.7887130468 & 0.0001640935 \\
\hline H & 1.2075072775 & 1.9583822837 & -0.0000449049 \\
\hline H & -2.0494623855 & 2.9196886511 & 0.0004526531 \\
\hline H & 0.2425347719 & 3.8642679751 & 0.0002172601 \\
\hline C & 2.2146735179 & 2.3550970843 & -0.0001685903 \\
\hline H & 0.6543208368 & -2.2736488139 & -0.0001906249 \\
\hline C & 0.5018716831 & -3.3469573225 & -0.0001617744 \\
\hline C & 2.1946794814 & -0.3407862571 & -0.0004095447 \\
\hline H & 1.9466555237 & -1.7507504031 & -0.0003311235 \\
\hline O & 2.8129099819 & -2.4029467621 & -0.0004070144 \\
\hline & 3.3634390923 & 0.1142790395 & -0.0002825337 \\
\hline
\end{tabular}

$$
\begin{aligned}
& \mathrm{PN}^{\bullet} \\
& \mathrm{E}_{0}=-575.297935 \\
& \mathrm{H}=-575.287578 \\
& \mathrm{G}=-575.333160 \\
& \mathrm{~T} 1=0.041 \\
& -1,2
\end{aligned}
$$$$
\text { C }
$$$$
-1.7887652352
$$$$
-1.9390289709
$$$$
0.0001661328
$$ 


\begin{tabular}{|l|l|l|l|}
\hline C & -0.4742276846 & -1.4281145019 & 0.0000872418 \\
\hline C & -0.273411023 & -0.0106519139 & 0.0001348281 \\
\hline C & -1.4100918353 & 0.8615483285 & 0.000250857 \\
\hline C & -2.7110040629 & 0.297119466 & 0.0003523515 \\
\hline H & -2.8841074427 & -1.0815411504 & 0.0003200203 \\
\hline C & -1.9347584306 & -3.0147004916 & 0.0000913423 \\
\hline C & 1.0401262569 & 0.5376639589 & 0.0000705725 \\
\hline H & -1.1922605821 & 2.2616775674 & 0.0002286919 \\
\hline H & -3.5697906264 & 0.9602190518 & 0.0004414884 \\
\hline C & -3.8874898079 & -1.4951744648 & 0.0003799399 \\
\hline C & 0.0959787534 & 2.777151764 & 0.0001077968 \\
\hline H & 1.2038328711 & 1.933639033 & 0.0000375489 \\
\hline H & -2.0511246563 & 2.9252064539 & 0.0002973077 \\
\hline H & 0.2412826402 & 3.8528953967 & 0.0000969568 \\
\hline C & 2.2116659595 & 2.3303943274 & 0.0000026533 \\
\hline H & 0.6778158381 & -2.2639951769 & -0.0001903802 \\
\hline C & 0.5294355337 & -3.3405644304 & -0.0004077157 \\
\hline C & 2.2213749097 & -0.336283518 & 0.0001451766 \\
\hline H & 1.9507017314 & -1.7484088088 & -0.0002656974 \\
\hline O & 2.8110607814 & -2.4106576461 & -0.0005959057 \\
\hline & 3.3882601113 & 0.1318797259 & -0.0008152076 \\
\hline
\end{tabular}

$$
\begin{aligned}
& \mathrm{PN}^{\bullet+} \\
& \mathrm{E}_{0}=-574.949751 \\
& \mathrm{H}=-574.939294 \\
& \mathrm{G}=-574.985143 \\
& \mathrm{~T} 1=0.038
\end{aligned}
$$




\section{1,2}

\begin{tabular}{|l|l|l|l|}
\hline C & -1.7704316555 & -1.9318371057 & 0.0001157627 \\
\hline C & -0.4521424871 & -1.4093143721 & 0.0000039698 \\
\hline C & -0.2604474025 & 0.0126328189 & 0.0000608318 \\
\hline C & -1.3895924007 & 0.8693224529 & 0.0002383824 \\
\hline C & -2.6795106641 & 0.3002854614 & 0.0003510029 \\
\hline H & -2.8627140211 & -1.0923503011 & 0.0002860685 \\
\hline C & -1.9032391292 & -3.0078554505 & 0.0000599296 \\
\hline C & 1.024346775 & 0.563750797 & -0.0000720851 \\
\hline H & -1.1976441537 & 2.2655709943 & 0.0002876542 \\
\hline H & -3.5435040539 & 0.9571908315 & 0.0004800817 \\
\hline C & -3.8655992087 & -1.4994728198 & 0.000370623 \\
\hline C & 0.0841582082 & 2.7977622358 & 0.0001604843 \\
\hline H & 1.1905119273 & 1.9521817713 & -0.0000197847 \\
\hline H & -2.064762868 & 2.9174088528 & 0.0004213535 \\
\hline H & 0.226268457 & 3.8708358377 & 0.0001968644 \\
\hline C & 2.1954844032 & 2.3591257408 & -0.000130697 \\
\hline H & 0.673046094 & -2.2838393053 & -0.0001653376 \\
\hline C & 0.4946741171 & -3.3535118611 & -0.0001794484 \\
\hline C & 2.2029215288 & -0.3412258622 & -0.0002990732 \\
\hline H & 1.9413724903 & -1.791392264 & -0.0003175782 \\
\hline O & 2.8082667218 & -2.4418070403 & -0.0004552495 \\
\hline & 3.343041322 & 0.0868125878 & -0.0004577552 \\
\hline
\end{tabular}

